\title{
TREE GROWTH REGULATORS: ISSUES OF CONTROL, MATTERS OF MANAGEMENT
}

\author{
by Gregory M. Moore
}

\begin{abstract}
All aspects of tree growth and development are regulated by plant hormones and other growth-regulating substances. The most common of these compounds are the auxins, gibberellins, and cytokinins, but other substances such as ethylene and abscisic acid also have a significant impact on tree biology. Usually there is a complex interaction between the growth regulators to affect physiological responses. An understanding of plant growth regulators can significantly increase the capacity of arborists to effectively manage trees in urban landscapes. However, to effectively use plant growth regulators as a tool for tree management, arborists must understand the subtlety of their interactions. Furthermore, to achieve arboricultural objectives, arborists must also understand the nature of the biochemistry and importance of precise and targeted application of plant growth regulators. In an era of modern arboriculture practice, tree hormones and growth regulators are not just substances of control but tools of effective tree management.
\end{abstract}

Biologists have recognised for over a century that plant growth and development is controlled by chemicals produced within the plant. Early work by German botanists such as Sachs was strongly influenced by work being done by animal and medical scientists on animal hormones at the time (Salisbury and Ross 1992). Consequently, plant physiologists adopted the following definition of a plant hormone: A plant hormone is an organic compound synthesised in 1 part of the plant and translocated to another where, in very low concentrations, it causes a physiological response (Moore 1984; Salisbury and Ross 1992).

Unfortunately, while this definition works well in human and animal biology, it is far from appropriate in plant biology, in which the sites of synthesis, transport, and response may all occur in the same tissue (Noggle and Fritz 1983). This definition is very restrictive and hardly practicable. Accordingly, for the purpose of this paper the following definition is proposed: A plant hormone is an organic compound synthesised within the plant and which may be transported to another site, or which may be active at the site of synthesis, which in very low concentrations causes a physiological response.

Generally speaking, in dealing with tree biology and management, the following groups or substances are considered to be the major hormonal groups that have an impact on plant growth and development: auxins, gibberellins, cytokinins (sometimes called kinins), abscisic acid (ABA), and ethylene. There has been considerable debate (Canny 1981, Street and Opik 1984, Moore 1984) about whether there are sufficient hormones to control the more than 2,000 aspects of plant growth and development that are identified by plant physiologists, and by what physiological mechanisms the hormones influence plant biology.

According to the definition, only naturally occurring compounds can be described as plant hormones. However, manufactured chemicals that mimic the structures and functions of hormones can also have significant effects on plant growth and development. Such substances are usually called plant growth regulators (or substances) and are often structural analogues of hormones (Figure 1). Plant growth regulators have been more widely used in horticulture than hormones have been.

\section{Mechanisms of Hormone Action}

Most plant hormones are active at very low concentrations, with many being active at a level of about $10^{-6} \mathrm{M}$, but it is not uncommon for hormones to be active at concentrations as low as $10^{-13} \mathrm{M}$. Plant tissues are particularly sensitive to hormone concentrations and respond quite differently to different concentrations. Thus, it is possible that a relatively small group of plant hormones can control a very wide range of plant growth and development phenomena when the following ranges of interactions are considered. 


\begin{tabular}{|ll|}
\hline Plant Hormones & Plant Growth Regulators \\
\hline Auxins & Auxins \\
Indoleacetic acid (IAA) & Naphthalene acetic acid \\
Phenylacetic acid (PAA) & (NAA) \\
4-chloro-indoleacetic Acid & $2,4-D$ \\
(4-Chl IAA) & 2,methyl-4- \\
Indoleacetonitrile (IAN) & chlorophenoxyacetic \\
Indoleproprionic acid (IPA) & acid (MCPA) \\
Indolebutyric acid (IBA) & $2,4,5-T$ \\
& \\
& \\
Gibberellins & \\
Gibberellic acids, 1 > 100 & \\
Cytokinins & Cytokinins \\
Zeatin, Isopentenyl adenine & Kinetin, Benzyladenine \\
Zeatin riboside & \\
Abscisic Acid & \\
Ethylene & \\
Others & \\
Fusicoccin, Brassins, Turgorins \\
Salicyclic Acid, Jasmonic Acid \\
Batasins
\end{tabular}

Figure 1. Major plant hormones and plant growth regulators.

- Different hormones affect different physiological processes in various plant tissues.

- Responses to hormones vary among trees.

- Different hormone concentrations effect different responses in a tissue.

- Two or more hormones can interact to stimulate different responses.

- The physiological state of a plant or tissue affects the response.

This combination of variables and interactions provides an almost infinite range of variation, and so it is possible that all aspects of plant growth and development can be controlled by the 5 groups of chemicals listed previously. It is also important to remember the major effects that the concentration of these compounds has on a tree's responses.

The precision of control that hormones can exert is enhanced by the fact that some hormones at particular concentrations promote growth, while others inhibit it. Indeed, a model can be constructed based on 4 kinds of generic regulatory chemicals (Coder 1994). These are growth promoters, growth inhibitors, environmental sensors, and reproduction initiators (Coder 1984; Moore 1984). The concepts of production, degradation, and antagonistic action allow for fine control of growth and development.

Given the sensitivity of plant tissues to low hormone concentrations, it is important to note the continuous metabolism of hormone synthesis and destruction. The destruction of hormones is essential in order that very high concentrations do not gradually accumulate in tissues. Hormones are readily biodegradable, not only by the plant's own internal biochemical processes but also by such organisms as bacteria. Furthermore, some hormones-such as the auxins-are quite sensitive to such environmental factors as light (Ridge 1991; Coder 1994).

The mechanism of the interaction between plant metabolism and plant hormones is not fully understood (Street and Opik 1984), but it is now recognised that plant hormones do affect gene activity (Ridge 1991). Whether the hormones directly affect the DNA or exercise control through an influence on mRNA is still not clear (Salisbury and Ross 1992). However, it is clear that an impact on the nucleic acids would explain how hormones influence such a diverse range of plant activities.

\section{Tree Hormones and Growth Regulators}

Auxins. Auxins are perhaps the best known and most significant group of plant hormones. They have been of interest to plant scientists since the 1880s and are known to affect a wide variety of plant biological activities. The most common hormonal auxin is indoleacetic acid (IAA), but other lesser-known auxins such as indoleproprionic acid (IPA) and 4-chloro-indoleacetic acid (4-ChI IAA) are not uncommon in plants and are more active than IAA.

The best-known auxins that are plant growth regulators are naphthalene acetic acid (NAA) and indolebutyric acid (IBA), which are widely used in the nursery industry, and substances such as the well-known herbicides 2,4-D and 2,4,5-T. In recent years, IBA has also been found to occur naturally in plants and is now regarded as a hormone (Salisbury and Ross 1992). The major effects of auxins on plant growth and development 


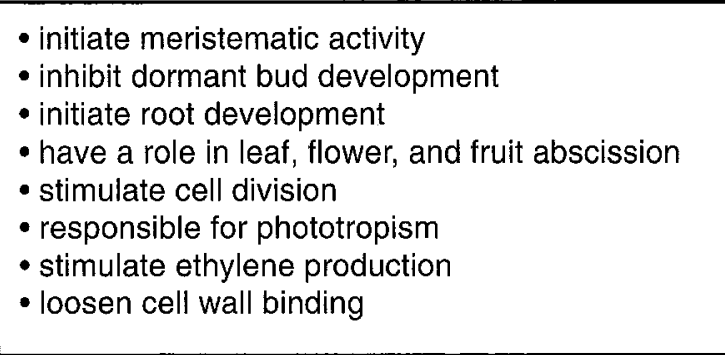

Figure 2. Major effects of auxins on tree biology.

are summarised in Figure 2. The role of NAA and IBA as rooting compounds is also important in plant production processes.

Hormonal auxins are easily biodegraded not only by the tree's metabolism but by other organisms. Hormonal auxins are easily and quickly degraded by light. In contrast, plant growth regulator auxins are much more stable and, as a consequence, can be prepared and used over a long period of time. Many of the effects of auxins on tree biology can be explained by their degradation in light and the subsequent effect that a lowered auxin concentration has on plant tissues. Compounds such as triiodobenzoic acid (TIBA) and naphthylthalamic acid (NPA) interfere with auxin transport (Salisbury and Ross 1992); these compounds are sometimes called antiauxins.

The role of auxins in tree biology. Auxins can have a significant impact on tree structure and function. Indeed, the range of effects is so broad that it is beyond the scope of this paper to consider them all. However, a number of the more significant effects of auxins that influence the management of trees will be discussed.

Auxins are produced in apical meristems, as well as in other rapidly growing and expanding plant tissues, such as young leaves. The auxins tend to move from these sites towards the base of the tree and, as they move, they affect the development of other plant tissues. Auxins are known to inhibit the development of dormant buds such as auxiliary, epicormic, and lignotuberous buds. Expansion of these buds is normally inhibited by auxins produced in the apical meristems and vigorous healthy leaves. When a tree is severely pruned (or worse, lopped) the growing tips and most, if not all, of the leaves are removed.
The internal hormonal balance is significantly disrupted, and for sometime after such an action, tree growth may be substantially unregulated and numerous shoots may develop from the previously dormant buds. The removal of auxin while relatively high levels of cytokinin are still produced from the roots is a significant cause of this imbalance.

The light sensitivity of hormonal auxins has a number of consequences for tree growth and development. This light sensitivity is well exemplified in the phototropic responses of many plants. In young trees that are staked, it is often observed that the tree grows away from the stake. It does so in many instances because the shade of the stake allows auxin levels to become higher nearer the stake than they are on the side of the tree away from it. Consequently, it is important to place a stake (should it be necessary) where it will have the least impact on the biology of the tree. In the southern hemisphere, the stake should always be placed on the south side of the tree, which will be shaded in any case. In the northern hemisphere, the stake should be placed on the north side of the tree.

Sometimes when a mature tree is removed, the trees that remain may suddenly begin sprouting along their trunks or large branches-usually because higher levels of light penetrate the disrupted canopy. This light degrades auxins, thereby removing the inhibition that had previously kept buds dormant. In some instances, such sprouting can be a nuisance and cause tree management problems, but in others it may be a positive outcome that rapidly fills an empty space within the canopy.

The oval shape that often develops in very large limbs is due to the formation of reaction wood. Reaction wood increases the cross-sectional area of the limb and is essential if it is to remain structurally sound. The production of reaction wood results from the differential concentrations of auxins across the branch (Coder 1994).

The use of plant growth regulator auxins such as IBA and NAA as root treatments following planting or transplanting is often recommended. These synthetic auxins are usually far more stable than hormonal auxins and are not degraded easily 
by light. Their use as rooting stimulants has been widely reported and in some situations they can be quite effective in improving success rates of plant establishment.

Gibberellins. Gibberellins were first discovered in Japan during the 1930s. A fungal disease of rice caused the plants to grow very tall and the heads to then fall under their own weight into the rice paddies, causing a significant reduction in grain production. It was subsequently discovered that the rice was a dwarf variety, which was infected by the fungus Gibberella fujikuroi. The fungus produced a substance that caused the internodes of the dwarf rice to elongate dramatically; that substance was called gibberellin (Salisbury and Ross 1992).

The gibberellins are variations of gibberellic acid (GA), an organic acid. Most plants contain only a few of the more than 100 GAs so far identified; the most commonly occurring is $\mathrm{GA}_{3}$. Young leaves are a major site of gibberellin synthesis, and the role of young leaves in tree canopy management is not well understood (Salisbury and Ross 1992). The most significant effect of $G A$ is the control of cell elongation and internode length, but it also has a number of other growth-promoting effects, many of which are similar to the effects of auxins (Figure 3).

Gibberellins are sometimes used to overcome bud or seed dormancy, especially in plants that require cold treatments such as stratification or vernalisation. They may also be used to delay senescence of leaves and fruit-particularly in citrus species-and to increase the berry size in some grape varieties.

The role of gibberellins in tree biology. The discovery of gibberellins provided an explanation for mutant dwarf varieties of trees and other

\footnotetext{
- stimulate cell elongation

- increase internode length

- induce flowering in some plants

- break some plant dormancies

- enhance geotropic responses

- cause production of seedless fruits
}

Figure 3. Major effects of gibberelins on tree biology. plants. Many dwarf varieties arise as a result of relatively simple mutations that are transmitted according to classical Mendelian genetics. The mutation that causes dwarfing blocks the production of gibberellic acid (Ridge 1991) and, as a consequence, internode length is much reduced.

Until recently, there has been little place for the use of gibberellins in tree management. Gibberellins are usually produced biologically and are expensive to buy, especially when they are likely to have little impact on target plants. However, there has been a widespread interest in the use of anti-gibberellins, which reduce the natural production of gibberellins within plants.

Chemicals such as ancymidol and cycocel (CCC) have been known for many years to inhibit gibberellin synthesis (Moore 1984; Ridge 1991; Salisbury and Ross 1992) and, at high concentrations, they can be lethal to plants. The modern anti-gibberellins, paclobutrazol and flurprimidol, are effective in decreasing gibberellin production and reducing internode lengththereby slowing the growth of trees. These inhibitors of gibberellin synthesis have significant potential as so-called "chemical pruners," and they have been widely tested in urban areas by utility companies that provide electrical services via overhead cabling. The application of an antigibberellin can induce a 3-to-7-year reduction in internode elongation and therefore increase the pruning cycle (Coder 1994).

The difficulty with the application of plant hormones and growth regulators is that concentrations must be accurately determined and the chemicals strictly targeted in their application. Should concentrations be miscalculated or should the chemical contact nontarget specimens, the effect can be both widespread and devastating. Indeed, this has been a limiting factor in the use of plant hormones and growth regulators in plant and tree management. Too often, people lack the training and sophistication to use such chemical tools adequately.

Cytokinins. Cytokinins were first identified as substances that could stimulate animal cell division (cytokinesis) and that had a similar effect on some plants. Kinetin, which had been isolated from the DNA of herring sperm, was the first iden- 


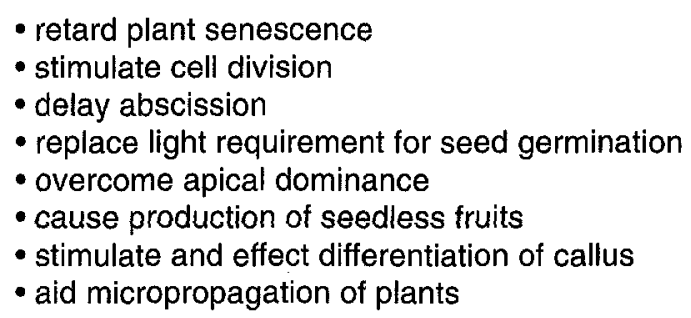

Figure 4. Major effects of cytokinins on tree biology.

tified cytokinin and is often described as a plant hormone. However to date, kinetin has never been isolated in plants and should not be described as a plant hormone. The best-known hormonal cytokinin found in plants is zeatin, which was first found in corn but has subsequently been identified in many other species. About 200 hormonal and synthetic cytokinins have been investigated (Salisbury and Ross 1992).

Cytokinins are a group of growth promoters that particularly stimulate cell division and expansion but also have a variety of other effects (Figure 4). In general terms, the auxins, gibberellins, and cytokinins can be described as plant growth promoters because they seem to have a positive effect at many concentrations on the growth of cells, tissues, and entire plant.

Another spectacular effect of cytokinins is the retardation and, in some instances, apparent reversal of the process of senescence. Not only are the degradation processes that accompany senescence delayed, but in some instances there is a genuine reversal that marks a rejuvenation. Such a rejuvenation involves the renewed production of chlorophylls and proteins and the redirection of the plant transport system. It is worth noting that some fungal diseases produce a symptom called the "green island effect," which is due to the production of cytokinins by the fungi, resulting in bright green areas in otherwise chlorotic and senescing leaves (Agrios 1988; Salisbury and Ross 1992).

The role of cytokinins in tree biology. The cytokinins have impressive effects on plant growth and development, but there is an inherent danger in their use. Any chemical that stimu- lates cell division and affects such fundamental processes as protein synthesis must be identified as a potential carcinogen. Although the importance of cytokinins in tree biology is recognised, they have been rarely used in aspects of tree management.

One of the most common uses of cytokinins is in the micropropagation of trees, especially through tissue culture (Ridge 1991; Coder 1994). Trees can be propagated and large numbers of clones of valuable varieties produced in a relatively short time using appropriate concentrations of auxins and cytokinins to stimulate differentiation from callus cells. Without growth regulators, callus tissue will not differentiate. The propagation of stressresistant and disease-resistant varieties of trees has become a useful management practice. Although not widespread, the technique has been used for producing phytophthora-resistant and stress-tolerant eucalypts (Eldridge et al. 1993).

Fasciation is a symptom of disease in some plants, in which stems become flattened and numerous lateral buds develop into branches that sometimes fuse together. The resultant structure is often like a witch's broom and is thought to be due to the bacterium Cornebacterium fascianas producing cytokinins that affect the plant's development (Salisbury and Ross 1992). It is probable that other pathogens that cause witch's brooms do so through the production of cytokinins. The only effective treatment of witch's brooms is careful pruning and removal.

The reversal of senescence caused by cytokinins has been experimentally used to increase the shelf life of such leafy vegetables as lettuce and cabbage, but this use has not been licensed commercially. Perhaps the best-known and most far-reaching use of cytokinins at the moment is as a component of tissue culture media. This application has widespread implications for tree breeding and production programs.

Abscisic acid (ABA). As its name indicates, abscisic acid has a role in the abscission of leaves, flowers, and fruit. The name is misleading, however, because ABA plays a much wider role in plant biology (Figure 5) through its effects on dormancy, stomatal behaviour, and stress physiology. Unlike the other hormones discussed in this article, ABA 
- induces dormancy

- causes leaf senescence

- promotes leaf, flower, and fruit abscission

- Inhibits seed germination

- may inhibit or promote flowering

- produced in response to environmental stress

- stimulates horticultural hardening off

Figure 5. Major effects of abscisic acid on tree biology.

is a single chemical compound. It is synthesised via a metabolic pathway, the beginning of which is shared with the production systems of gibberellins and the carotenoid pigments.

Unlike auxins, gibberellins, and cytokininswhich are generally seen as growth-promoting substances-ABA seems to be an effective inhibitor of plant growth. It is involved in the dormancy of various plant tissues and has a role in the senescence and abscission processes. Many of the effects of ABA can be countered by the application of auxins or gibberellins. Thus, the inhibitory role of ABA counteracts the stimulatory role of the growth-promoting hormones. The relationship between concentrations of growth promoters and inhibitors provides a mechanism for very fine control of plant growth and development.

The mechanism of ABA inhibition is still uncertain. However, it appears to inhibit RNA and protein synthesis and clearly influences enzyme activity. It also has the capacity to affect membrane permeability and it is through this effect that its influence on stomatal behaviour is exerted.

The role of abscisic acid in tree biology. Studies over the past 2 decades have revealed that when whole plants or plant tissues are under stress, there tends to be an accumulation of $A B A$. This has led to ABA being described as the "stress hormone." Increases in ABA levels have been recorded in plants after drought, flooding, nutrient deficiencies, and even mechanical injury. The increase in ABA level appears to be part of the system that allows plants to respond and adapt to stress. This aspect of ABA behaviour should prove useful in tree management.

For many years, horticulturists have been aware of the phenomenon of "hardening off." This process is an inherent part of most plant propagation systems and involves exposing plants to moderate levels of environmental stress with a view to preparing them for planting and subsequent establishment. It has been suggested that the hardening-off process stimulates $A B A$ production, and that it is the $A B A$ that enhances survival and establishment rates. However, research has been disappointingly inconclusive. The possibility of replacing the hardening-off process with a single application of ABA is worthy of further investigation and is currently the subject of a research project at Burnley College, Australia.

It has also been suggested that the use of $A B A$ may be an effective management technique for improving survival and establishment rates when trees are planted from tube or container stock. The suggestion that the use of ABA before planting may reduce the impact of transplanting shock has been investigated (Trezise 1994), but the results were inconclusive and further research is continuing. The possibility of reducing the impact of transplanting stress for small and large trees seems real, and further research should improve effective management strategies.

Ethylene. Ethylene is an interesting plant hormone, which once again does not conform to the conventional definition because it is a volatile substance that is never translocated internally. Consequently, some physiologists regard ethylene as a plant growth regulator rather than a hormone.

The production of ethylene in a variety of plant tissues such as flowers, leaves, fruits, roots, and seeds is well known, and its impact on fruit storage and transport has been recognised for over a century. Many of the early studies on ethylene focused on the impact of coal gas on street trees, the promotion of flowering, and the storage of fruit. Indeed, there were some disastrous explosions in ships as a result of ethylene accumulation during the transport of crops such as apples.

The diverse range of effects caused by ethylene (Figure 6) suggests an association with other hormones such as cytokinins and ABA but especially with auxins. Indeed, the relationship between auxins and ethylene is so strong that there is still some confusion about whether the effect is a result of ethylene or auxin levels. Within the 


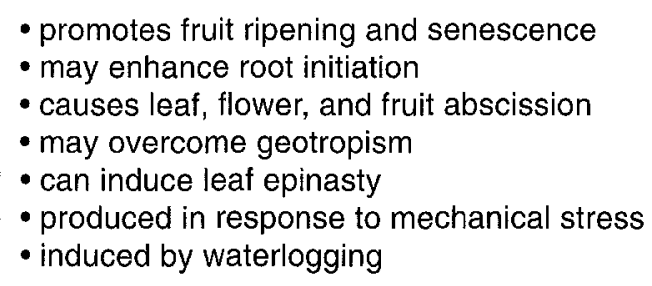

Figure 6. Major effects of ethylene on tree biology.

plant, ethylene seems to be produced from a simple reaction involving the amino acid methionine. The ethylene then binds-probably to copper in an enzyme-and may be important in the processes of abscission and senescence.

The role of ethylene in tree biology. Ethylene is known to induce flowering in mangoes and many of the bromeliaeds, but it also inhibits the elongation of roots, stems, and leaves in some species. The inhibitory effects of ethylene are also demonstrated in its induction of epinasty and its production in response to wounding or infection. Ethylene also has a major role in the abscission and senescence processes of many trees.

In an urban context, it is possible to manipulate some of the shedding and retention processes in street trees through the application of ethylene-producing substances. For example, it is possible to use ethylene-producing foliar sprays such as Ethrel (which contains ethaphon) on deciduous tree species to cause a timed and coordinated shedding of leaves in autumn. This can reduce street sweeping and cleaning costs by up to $75 \%$. A similar approach may be used to time ripening and shedding of some fruits, which may be an advantage in an urban context if the fruits are undesirable or pose potential hazard. Florel, which also contains ethaphon, is used to stimulate flower abscission, which then prevents fruit formation.

\section{Hormone Interactions and Tree Management}

Many aspects of plant growth and development result from the interaction of 2 or more plant hormones rather than the influence of a single substance or hormone group. Many of these interactions are poorly understood and very complicated, but a few that have implications for ur- ban tree management are worthy of further exploration.

Tree leaf abscission and retention. One of the best-known examples of hormonal interactions involves the retention of young expanding leaves and the abscission of mature senescing leaves. Although the process is not fully understood (Figure 7), it is believed to involve the interaction of at least 4 different hormones (Moore 1984; Salisbury and Ross 1992). Furthermore, it illustrates the importance both of the interaction of these hormones and the changes their concentrations can bring in plant growth and development.

Tree transplanting. Similarly, there is no interaction between auxins and cytokinins when a tree is transplanted. The newly transplanted tree often has significant root loss, especially of young root tips. This reduces cytokinin production but without altering auxin production in the foliage. The relatively high ratio of auxin to cytokinin stimulates root development (Coder 1994). The old practice of pruning off the foliage in such situations reduces auxin production and so delays the initiation of new roots and the subsequent recovery of the tree. Canopy pruning under such circumstances is an unacceptable arboriculture practice (Coder 1994).

Transplanting mature tree specimens is an established horticultural and arboricultural practice. Most of the principles involved in mature tree transplanting are well known. A simple flow chart for the management of the process can be used and recognises that an important aspect in the transplanting process is to maintain the same orientation with respect to a compass bearing (usually north) of the tree at all times. Properly re-establishing the tree requires an attention to site detail that takes account of the soil and light factors that influence tree growth and development (Pirone et al. 1988; Harris 1992).

This recommendation is based upon the recognition that one of the most important influences on the growth and development of a tree is its position in relation to the sun. This orientation influences the arrangement of the foliage and the development of the branching system. It also influences leaf bud burst, flowering sequence across the canopy, and the sequences of bark 


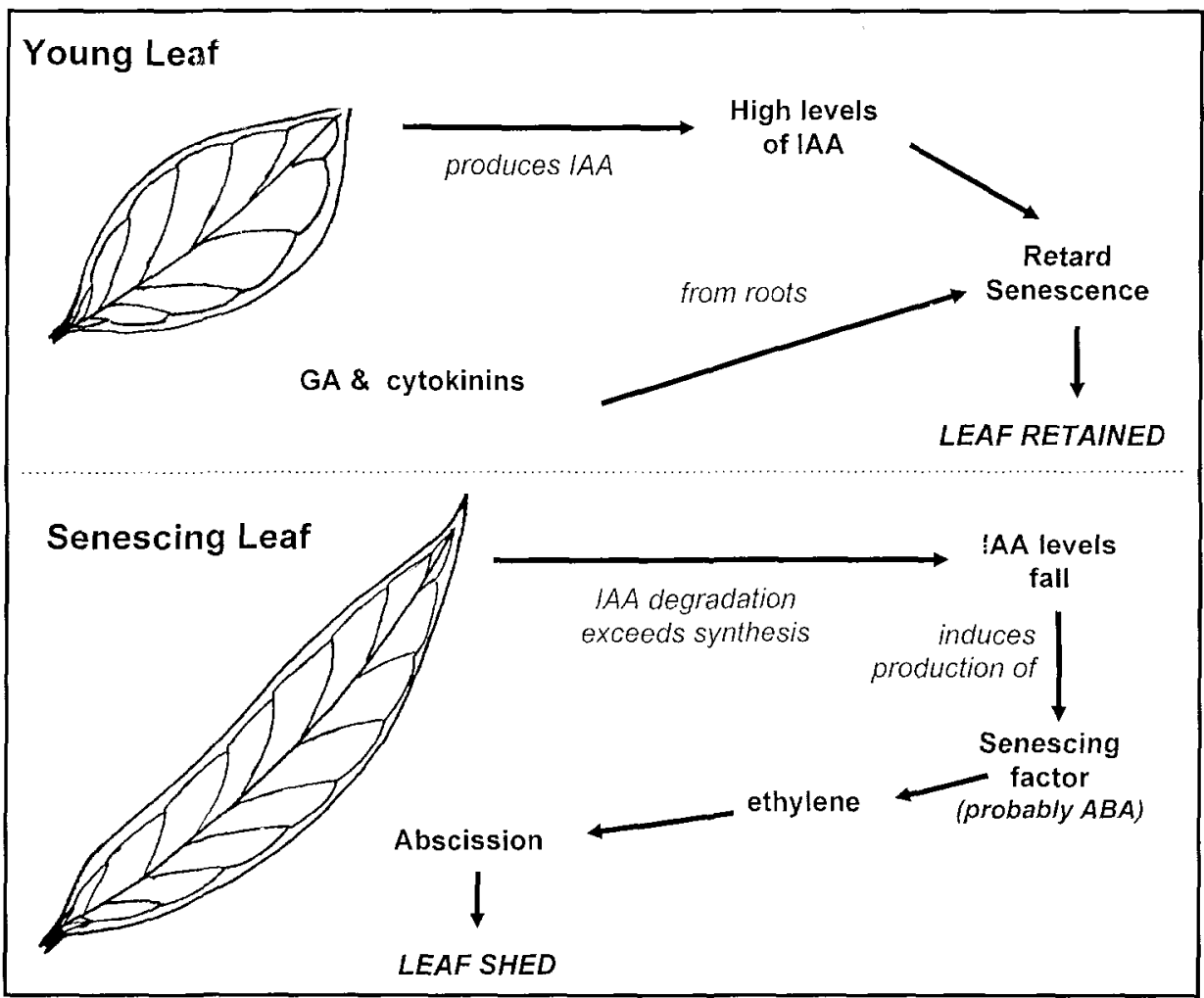

Figure 7. Hormones involved in leaf abscission.

shedding. Many, if not all, of these processes are influenced by the activities of plant hormones and need to be understood if transplanting is to be successful.

Careful and correct orientation of the tree during transplanting will preserve the relationships that the tree has in relation to the sun and light levels. Proper orientation should minimise the disruption that transplanting might cause to internal hormone levels, such as auxins, and thereby reduce transplanting shock and stress levels, resulting in a more rapid establishment of a healthy and vigorous tree.

Proper transplanting procedure also raises the possibility of applying root-promoting growth regulators such as IBA or NAA as a means of encouraging root growth. These substances may need to be applied on a regular basis after transplanting, and a cost-benefit analysis should be done to ascertain whether this is an effective management practice.
Tree pruning. As explained earlier, pruning and lopping of trees in such a way as to remove most of the foliage disrupts not only the level of auxins but of other hormones such as cytokinins as well. As a consequence of such a poor pruning practice, large numbers of dormant buds, particularly epicormic buds, may develop rapidly. They are often poorly attached but grow at rates of up to $30 \mathrm{~cm}$ (11.8 in.) in a week, or 5 to $6 \mathrm{~m}$ (16.4 to $19.7 \mathrm{ft}$ ) in a year under good growing conditions. Because of their weak attachment, the branches are easily shed and represent a significant hazard in some urban settings.

It is important to understand that by leaving some foliage on the tree, a measure of control is left in place that may provide a canopy more in line with the desired objectives of tree management. If all of the foliage is removed, then regular and ongoing maintenance of subsequent growth is essential and must be budgeted for as a matter of routine management.

\section{Conclusion}

Plant hormones and growth regulators can be used to more effectively manage trees in urban areas. Although the biology of these substances is not fully understood, their potential for improving the efficiency and effectiveness of urban tree management is substantial. This potential, however, is often unrealised because

- the hormones and growth regulators are expensive; 
- their use requires sophisticated and welltrained workers;

- there are difficulties in targeting species; and

- their activity at very low concentrations means that great care must be taken in their preparation and application.

Plant growth regulators have outstanding potential for arboricultural manipulation of urban trees. They are currently used in some contexts but require competent and professional workers. Furthermore, it is likely that direct application of hormones and plant growth regulators in many situations will provide an unacceptable environmental risk. It is likely that the desired tree responses will be genetically engineered into urban trees so that the responses can be manipulated at appropriate times by tree managers.

Acknowledgments. The author acknowledges the assistance of K.D. Smith, K.L. Blaze, and M. Looker in commenting upon and improving the content of this paper. Thanks also to G. Gay, K.D. Smith, and S. Miller for preparation of the document and diagrams

\section{Literature Cited}

Agrios, G.N. 1988. (3rd ed). Plant Pathology. Academic Press, New York, NY.

Canny, M.J. 1981. A systems approach to the control of plant development. Abstracts, XIII International Botanical Congress, Sydney, Australia.

Coder, K.D. 1994. Regulation of tree growth: Keeping the green side up. Arborist News, 3(1):33-36.

Eldridge, K., J. Davidson, C. Harwood, and G. van Wyk. 1993. Eucalypt Domestication and Breeding. Oxford University Press, New York, NY.

Harris, R.W. 1992. (2nd ed). Arboriculture: Integrated Management of Landscape Trees, Shrubs, and Vines. Prentice-Hall, Englewood Cliffs, NJ. 674 pp.

Moore, G.M. 1984. Mechanisms of hormone action in plants. International Plant Propagators Society, Proceedings 34:79-90.

Noggle, G.R., and G.J. Fritz. 1983. Introduction to Plant Physiology. Prentice-Hall, Englewood Cliffs, NJ.

Pirone, P.P., J.R. Hartman, M.A. Sall, and T.P. Pirone. 1988. (6th ed.) Tree Maintenance. Oxford University Press, New York, NY.

Ridge, I. 1991. Plant Physiology. Hodder and Stoughon/Open University, London.
Salisbury, F.B., and C. Ross. 1992. (4th ed.) Plant Physiology. Wadsworth, Belmont, CA.

Street, H.E., and H. Opik. 1984. The Physiology of Flowering Plants: Their Growth and Development. Edward Arnold, London.

Trezise, G. 1994. The role of abscisic acid (ABA) in the management of environmental stress. Horticultural Project Report. VCAH, Burnley, Australia. $59 \mathrm{pp}$.

\section{Burnley College \\ University of Melbourne \\ Yarra Boulevard \\ Richmond, Victoria, 3121 Australia}

